5. While the contraction of the fibres of the ciliary sphincter in the states above noted takes place uniformly all round, establishing a degree of general tonicity, a contraction of some of the fibres or their relaxation from fatigue relatively to the rest may take place in segments of the sphincter, and this may be-

A. Meridianal-that is, when the segments are at the opposite ends of a meridian of the lens, in which case contraction increases and relaxation diminishes the re. fractive power of the lens in this meridian only. Such variations in activity occurring in both hypermetropia or myopia may either-

(a) Correct regular astigmatism, contraction doing so when acting in the meridian of least curvature of the corvea and relaxation when acting in the meridian of greatest curvature of the cornea. In this way hypermetropic astigmatism may be corrected, and though this is stated by Eruest Clarke and others to be possible only up to $0.75 \mathrm{D}$, I liave recorded numerous cases of 2 and $3 \mathrm{D}$, two of $4 \mathrm{D}$, and two of $5 \mathrm{D}$ in this way fully corrected, an interesting feature of some of them being that this correction was unattended by any symptom of eyestrain, this applying to both cases of $4 \mathrm{D}$ noted. As on post-cycloplegic testing the cylinders found to correct the astigmatism in the static refraction were in some cases rejected, in others accepted, and in still others weaker ones were accepted, it is evident that, just as in the case of hypermetropia, we have to cousider manifest, latent, and total astigmatism, the amounts of which vary in different cases and are subject to variation in the same case. Auto-correction of a low degree of astigmatism is common long after the presbyopic age, and I lave notes of the correction of simple hypermetropic astigmatism of $0.75 \mathrm{D}$ at 58 years.

(b) Cause regular astigmatism, in which case the segments may vary from time to time, necessitating corresponding changes in the axis of the correcting cylinder.

B. Non-meridianal-that is, not corresponding to one of the chief meridians of the lens, when its refraction is increased eccentrically so that, either in the existence of corneal astigmatism or apart from it, irregular astigmatism -that is, not correctable by glasses-results with occasionally monocular diplopia.

6. At times, from the above causes-and this I have found up to the age of 58-one or both of the ciliary muscles may pass into a phase of "irritability" from which regular astigmatism nay be corrected or caused at intervals, and, if the latter, the meridian may be varied from day to day, while in either case the astigmatism may every now and then be rendered irregular, and then, if monocular diplopia is present, from variations in the segments the fainter object may appear displaced from the more distinct in different directions at different times Naturally the above abnormalities in the activity of the ciliary nuscles are a fertile source of asthenopia and are of special interest when found at an age at which they liave been for the most part unsuspected.

7. As to treatment, it becomes imperative in all cases of asthenopia in which spasm or irritability of the ciliary muscles may be the possible cause, apart from lessening as much as possible the near work and doing it in a good light screened from the eyes by a green shade, etc., to paralyse the ciliary muscles as above and estimate the static refraction; and if the cylinders found necessary are not accepted and weaker ones accepted are still productive of asthenopia and we do not fear glaucoma, to keep the accommodation thoroughly paralysed by atropin for several weeks while the cylinders correcting the total astigmatism are worn and general and nervous tonjc treatment carried out, when they will almost invariably be accepted-an alternative plan being to prescribe the cylinders correcting the total astigmatism for constant use, trusting to the corrective partial contraction of the cilia:y muscles giving way in time.

ST. ANDREW's COLLEGE, Toronto, is being converted into a military hospital, to serve as the chief demobilization centre for the whole of Canada. Twenty.five new buildings are to be erected, and when completed the institution will are to be erected, and when completed the institution will
cover twenty-flve acres of ground. Though not intended to be permanent, the hospital will be used for many years after the war for the treatment of invalided or incapaeitated soldiers.

\section{BLOOD PRESSURE IN PULMONARY TUBERCULOSIS.}

\author{
BY
}

RICHARD J. CYRIAX, M.D.BRUX., M.R.C.S., D.P.H.,

ASSISTANT RESIDENT MEDICAL OFFICER, MOUNT VERNON HOSPITAL, NORTHWOOD.

Is the Jocrisal of August 10th appeared an article by D1. E. F. Cyriax on blood pressure in unilateral war traumatisms, pointing out that the pressures in the two arms do not always coincide, and, furthermore, that the pressure may be higher in one arm than in the other on one day, and the exact opposite a few days later. He pointed out also that the actual readings were influenced neither by the arm from which the first reading was taken, nor by the interval between the two readings.

A somewhat similar phenomenon appears to occur in some cases of phthisis, whether unilateral or bilateralnamely, that the pressure in the two arms is by no means invariably the same, a source of serious error being thereby introduced into the whole question of blood pressure in this disease.

The following observations were made upon 19 cases under the care of Dr. T. N. Kelynack at the Mount Vernon Hospital, Northwood. The readings were taken at one sitting, first on one arm and then upon the other, as rapidly as was compatible with accuracy, with a Riva-Rocci instrument, by the auscultation method combined with palpation of the radial pulse. Both systolic and diastolic readings were taken, but it is especially to the former that I wish to direct attention at present. Differences of $5 \mathrm{~mm}$. or less have been disregarded in order to make every allowance for the rise induced by the constriction of the armlet.

Five patients, all males, were confined to bed, except where otherwise stated. Tubercle bacilli have been found in all their sputa. In two cases differences of more than $5 \mathrm{~mm}$. in the systolic pressures were not found; two ob. servations were made on each patient at intervals of some days. Both have lesions in both lungs.

R. B., aged 18. Inflitration right upper and apex right lower Septemoptysis 6 ounces September 18th

September 27th. Blood pressure: R. 121.68; J. 119.68.

(Systolic difference, $\mathbf{R} .+2=0$.)

October 4th. R. 136.83; L. 132.84. (R. $+4=0$ )

Sodium nitrite, one grain, administered four times daily.

October 9th. R. 115.79; L. 113.78. (R. $+2=0$.)

October 11th. R. (taken first) 118.94; L. 132.78. (R. - 14.)

October 12th. R. 114.82; L. (taken first) 131.80. (R. - 17.)

The systolic pressure is thus lowest at present upon the diseased side. Sodium nitrite is still being administered.

\section{CASE IV.}

W. J. B., aged 41 . Infiltration both upper lobes, principally on the left.

September 27th. R. 124.84 ; L. 120.84. (I. $-4=0$.)

October 7th. Dr. Kelynack considered that a fresh spread was probably taking plaee. October 9th. Dr. Kinton noticed hat the principal activity was on the left side.

October 10th. R. (taken first) 102.85; L. 116.80. (L. + 14.)

October 12th. R. 124.88 ; L. (taken first) 136.90. (L. + 12.)

The systolic pressure is thus higher upon the side which is most active at present.

H. J. W., aged 47. Infiltration both upper lobes, principally upon the left, scattered foci throughout.

October 2nd. R. 105.80; L. 101.80. (L. $-4=0$.)

October 4th, 5th, 6th. Patient rested up two hours.

October 7 th. Sputum stained. Rest in.bed.

October 10th. R. 128.84; L. (taken first) 114.82. (L. - 14.)

October 12th. Patient rested up two hours. 'I'wo hour after return to bed, R. (taken first) 116.82; I. 109.83. (L. - 7.) All the above readings were taken while the patients were n bed.

In the remaining 14 cases, not confined to bed, differences in the systolic pressure of more than $5 \mathrm{~mm}$. were found in 6 . All six are cases of bilateral disease; tubercle bacilli have been found in all their sputa. The differences amounted, in the male patients, to 7,8 , and $13 \mathrm{~mm}$. respectively; in the female patients to 14,8 , and $10 \mathrm{~mm}$

I am convinced that the differences cannot be die to the constriction of the armlet. If they were so due, the same relation'slip ought invariabiy to subsist between the reading taken from the first and that taken from the second arm. No such relationship has been found. 
At present I refrain from suggesting an explanation, vither of the phenomenon itself, or of the latter's clinical significance. It may nevertheless be stated, as far as the observations made up to the present time enable an opinion to be formed, that the systolic pressure in the arm appears to be lower, as a general rule, upon the diseased side in a unilateral, upon the most diseased or most active side in a bilateral, lesion. Further investigations are in active progress.

This possible source of fallacy in estimating the blood pressure in cases of pulmonary tuberculosis is too obvious to need further emplasis.

I am greatly indebted to Dr. Kelynack for his kind permission to publish these preliminary observations, and to $\mathrm{Dr}$ Kinton, Medical Superintendent, for his encouragement and assistance.

\section{Alemwarand : \\ MEDICAL, SURGICAL, OBSTETRICAL.}

\section{PREVENTION OF INFLUENZA IN NAVAL} AMBULANCE TRAINS

IN view of the many and varied suggestions put forward for the prevention of influenza, the following notes of the means taken to prevent infection in the case of the crews of four naval ambulance trains which were used to convey a number of cases of influenza in an acute stage on four different occasions, may be of interest:

On June 29th Naval Ambulance Train. No. 1 proceeded to Dover from Chatham to entrain 104 cases of influenza for
transport to the Royal Naval Hospital, Chatham. The train transport to the Royal Naval Hospital, Chatham. The train was at Dover for four hours and seven minutes, during and brought on board. The train left Dover at 12.30 p.m. and reached Gillingham for Royal Naval Hospital, Chatham, at $1.51 \mathrm{p} . \mathrm{m}$. Some of the crew of this train were in contact with bad cases of influenza for nearly five hours. The crew of the train consists of 37 sick berth and sick berth reserve ratings and two medical officers, and all were given quinine bisulphate gr. 5 , and used a gargle of eusol. All bedding was sterilized and the train was washed down inside with a weak solutic a of izal after the run. No case of influenza developed amongst the crew or officers of this train.

On July 10th Naval Ambulance Train No. 3 proceeded to On July 10th Naval Ambulance Train No. 3 proceeded to pover from Chatham to entrain 140 cases of influenza. This train arrived at Dover 2.23 p.m., left again at 5.20 p.m., and reached Gillingham at 6.47 p.m. Cases from various sources were being taken on board during the whole time the train lay at Dover. Rather less than half the cases would be described lance Train No. 1, the crew consisted of 37 sick berth and sick berth reserve ratings and two medical officers, and all were given quinine bisulphate gr. 5 , and gargled with $a$ weak solution of carbolic acid. Influenza did not attack any man or officer employed on this occasion.

On October 9th R.N. Ambulance Train No. 2 was dispatched to Gosport to convey 42 lascars, all of whom had a severe form of influenza, complicated in 6 crses with bronchopneumonia. The cases were entrained between 11.5 a.m. and 12.25 p.m. The the trin left Gopert for Gro When the train left Gosport for Greenwich. At Green wich the In this train all the crew and the medical officers took quinine bisulphate gr. 5, and they also gargled with a solution of potassium chlorate and carbolic acid. The train was disinlected with a solution of izal when it returned to Chatham. In this train, as in the other two, no case of inflnenza occurred in any officer or rating.

On October 31st Naval Ambulance Train No. 5 made a journey from Edinburgh to Stobhill via Queensferry and took up and carried a total of 108 cases of influenza. Most of the cases of influenza were on board the train for about four hours, but some were as long as six. In this case the officers and crew took quinine gr. 5 and gargled with a solution of potassium permanganate 1 in 5,000. The inside of the train was afterwards sprayed with izal and then washed down with carbolicene. No case of influenza occurred in the personnel of this train after this journey.

In the naval ambulance trains officers and men live continually on board. In all these four instances 39 healthy individuals were in close contact for several hours with a larger number of actively infective cases of influenza, and certain precautions were taken in each case, and not one of the 39 became infected with the disease. Quinine has always been considered by many as a prophylactic in influenza, and, combined with gargling of the fauces, certainly appears to hape been effective in the four instances cited. Doubtless the disinfection of the trains with antiseptic solutions also tended to "lessen the risk of infection.

Spraying of the nasopharynx or washing out the posteriot nares with some mild antiseptic would doubtless be addi tional safeguards, but these measures are not very easily carried out, and in view of the immunity of the 156 persons mentioned above who used antiseptic gargles they are apparently not absolutely necessary.

K. H. Jones, M.B., Surgeon Commander R.N., Naval Medical Transport Officer. Chathem.

FILARIASIS AMONGST AUSTRALIAN TROOPS. Is the article on filariasis amongst Australian troops Captain Rimmer, R.A.M.C. (October 12th, p. 405), makes note of four cases amongst Queenslanders occurring at Havre. As I was born, bred, and practised in Queens land, some points which have come to my knowledge may be of interest to Captain Rimmer.

In Queensland generally, and particularly in the north filariasis is extremely common. As a record of its fre quency I may quote the instance of a pregnant woman with mastitis in whose blood the filarial embryos were found in great numbers. The inmates of that household numbered eight, and merely for the sake of interest my father and self examined the blood of these people, and in six out of the eight embryos were found without any trouble. The remaining two gave a negative result:

Filariasis, with its sequelae, is one of the most prevalent of Queensland diseases, and is one of those conditions which has always to be excluded in making a diagnosis The percentage of the inhabitants who, although they have the nematode in their blood, show no outward or visible sign of their condition must be astounding. Often times have we examined patients who have come to the consulting room for advice as to other diseases and found filarial embryos in the blood.

In these circumstances it can hardly bo wondered that : number of Queensland soldiers of the A.I.F., even though they have been away from their own country for somc length of time, are subject to acute exacerbations of filariasis.

The amount of literature on filaria in Queensland is fairly extensive; a great deal of it is to be found in past numbers of the Australian Medical Journal.

A. T. H. Nisbet, Captain A.A.M.C.

Ir might be of interest to state that microtilaria were found in 10 per cent. of the patients admitted to the Brisbane General Hospital. These statistics were compiled some few years ago from patients admitted to hospital suffering from various complaints. That hospital receives patients from all over Queensland, but more especially from the southern part, from Bundaberg south to the New South Wales border. From the figures the occurrence of filariasis amongst Queensland troops is probably about 10 per cent. Filariasis is practically never heard of in New South Wales, Victoria, and South Australia.
France.
A. M. LiLlex, Captain A.A.M.C.

INTESTINAL OBSTRUCTION AND APPENDICITIS.

IT is essential in cases of obstruction of the bowels to ask, "Have you ever had appendicitis?" and to remember: that, irrespective of the time, weeks or years, which may have intervened between an attack of this malady and the onset of obstruction, a three-inch exploratory incision should be made in the right semilunar line low enough just to expose the region of ileo-caecal valve, as the obstruc. tion-usually a kink with dense adhesions-will invariably be found in the last few inches of the ileum. Personal experience warrants the conclusion that the stereotyperl textbook central incision in such cases is attended with disastrous results, as in the search for the lesion the dis tended paralysed intestines are needlessly exposed and handled, with consequent shock. The vital factors in the procedure are to get directly to the spot, avoid deadly protrusion of bowels, straighten out the kink, and secure one end of a large drainage tube in the site of adbesions, and then rapidly close rest of parietal wound with a fow strong silkworm-gut sutures. With a funnel and long tube a rectal injection of a half-bottle of champagne 